\title{
SUBORDINATION BY UNIVALENT FUNCTIONS
}

\author{
SUNDER SINGH AND RAM SINGH
}

\begin{abstract}
Let $K$ be the class of functions $f(z)=z+a_{2} z^{2}+\cdots$, which are regular and univalently convex in $|z|<1$. In this paper we establish certain subordination relations between an arbitrary member $f$ of $K$, its partial sums and the functions $(\lambda / z) \int_{0}^{z} f(t) d t$ and $\mu \int_{0}^{z} t^{-1} f(t) d t$. The well-known result that $z / 2$ is subordinate to $f(z)$ in $|z|<1$ for every $f$ belonging to $K$ follows as a particular case from our results. We also improve certain results of Robinson regarding subordination by univalent functions. A sufficient condition for a univalent function to be convex of order $\alpha$ is also given.
\end{abstract}

Introduction. Let $A$ denote the class of functions $f(z)=z+a_{2} z^{2}+\cdots$ which are regular in $|z|<1$. We denote by $S$ the subclass of $A$ consisting of functions $f$ which are univalent in $|z|<1 ; S^{*}$ and $K$ will stand for the usual subclasses of $S$ whose members are, respectively, starlike (w.r.t. the origin) and convex in $|z|<1$. A function $f$ belonging to $A$ is said to be convex of order $\alpha, 0<\alpha<1$, in $|z|<1$ if and only if

$$
\operatorname{Re}\left(1+\frac{z f^{\prime \prime}(z)}{f^{\prime}(z)}\right)>\alpha \quad(|z|<1),
$$

and we shall denote by $K(\alpha)$ the class of functions satisfying (1).

A function $f$ is said to be subordinate to a function $F$ (in symbols $f(z) \prec F(z)$ ) in $|z|<r$ if there exists a regular function $w(z)$ with $|w(z)|<|z|<r$, such that $f(z)=F(w(z))$ in $|z|<r$. For $F$ univalent in $|z|<r, f(z)<F(z)$ in $|z|<r$ is equivalent to $f(0)=F(0)$ and $f(|z|<r) \subset F(|z|<r)$.

In the sequel whenever we come across the notation $f(z)<F(z)$ for $|z|<r$ we shall understand that the superordinate function $F$ is univalent in $|z|<r$ and $f(0)=F(0)$.

The Hadamard product or convolution of two power series $f(z)=\sum_{n=0}^{\infty} a_{n} z^{n}$ and $g(z)=\sum_{n=0}^{\infty} b_{n} z^{n}$ is defined as the power series $\sum_{n=0}^{\infty} a_{n} b_{n} z^{n}$ and denoted by $(f * g)(z)$.

A sequence $\left\{b_{n}\right\}_{1}^{\infty}$ of complex numbers is called a subordinating factor sequence if, whenever $f(z)=\sum_{n=1}^{\infty} a_{n} z^{n}$ is univalent and convex in $|z|<1$, we have

$$
\sum_{n=1}^{\infty} a_{n} b_{n} z^{n} \prec f(z)
$$

in $|z|<1$.

Received by the editors September 19, 1979 and, in revised form, February 25, 1980.

AMS (MOS) subject classifications (1970). Primary 30A32.

Key words and phrases. Regular functions, univalent, starlike and convex functions, subordination, subordinating factor sequences, Hadamard product. 
The de la Vallée Poussin means of a regular function $f(z)=a_{1} z+a_{2} z^{2}+\cdots$ are defined by

$$
V_{n}(z, f)=\left(\begin{array}{c}
2 n \\
n
\end{array}\right)^{-1} \sum_{k=1}^{n}\left(\begin{array}{c}
2 n \\
n+k
\end{array}\right) a_{k} z^{k}
$$

for $n=1,2,3, \ldots$ Pólya and Schoenberg [5, Theorem 2, p. 298] showed that these means are convex (starlike) if and only if $f$ is convex (starlike).

Robinson found a number $r_{0}, 0<r_{0}<1$, with the property that if $f$ and $F$ are regular for $|z|<1, f(0)=F(0)$ then the relation $z f^{\prime}(z) \prec z F^{\prime}(z)$ in $|z|<1$ implies that $f(z) \prec F(z)$ at least for $|z|<r_{0}$ [8, Theorem, p. 20]. In our first theorem we improve this result. Theorem 2 improves a similar result of Robinson [8, Theorem, p. 22]. In Theorem 3 we give a sufficient condition, involving the principle of subordination, for a univalent function to be convex of order $\alpha$. Theorems 4 and 5 deal with subordination relations between an arbitrary element $f$ of $K$ and its "convex transformations" $(\lambda / z) \int_{0}^{z} f(t) d t$ and $\mu \int_{0}^{z} t^{-1} f(t) d t$, where $\lambda, \mu$ are some positive real numbers. Theorem 6 generalizes the well-known result $z / 2 \prec f(z)$ for every member $f$ of $K$ and the last one deals with a subordination relation between the de la Vallée Poussin mean of second order and the second partial sum of a normalized convex univalent function.

The following result due to Suffridge [10] will be used to prove Theorem 1.

LEMMA 1. If $f \in K, g(z)=\sum_{n-1}^{\infty} b_{n} z^{n}$ is regular in $|z|<1$, and $z g^{\prime}(z) \prec z f^{\prime}(z)$ for $|z|<1$, then $g(z) \prec f(z)$.

THEOREM 1. If $f(z)$ and $F(z)$ are regular for $|z|<1, z F^{\prime}(z)$ is univalent in $|z|<1$, $f(0)=F(0)$ and $z f^{\prime}(z) \prec z F^{\prime}(z)$, then

$$
f(z) \prec F(z) \text { at least for }|z|<r_{0},
$$

where $r_{0}=\tanh \pi / 4=0.655 \ldots$

Proof. Since $z F^{\prime}(z)$ is univalent in $|z|<1$, it is starlike for $|z|<$ $\tanh \pi / 4=r_{0}$ (say) and hence $F(z)$ is convex in this disc. From this it follows that $F\left(r_{0} z\right)$ is convex in $|z|<1$. Thus our hypothesis implies that

$$
z f^{\prime}\left(r_{0} z\right) \prec z F^{\prime}\left(r_{0} z\right), \quad|z|<1,
$$

where now $F\left(r_{0} z\right)$ is convex in $|z|<1$. From this, using Lemma 1, we conclude that

$$
f\left(r_{0} z\right) \prec F\left(r_{0} z\right)
$$

in $|z|<1$ and hence

$$
f(z) \prec F(z)
$$

at least for $|z| \leqslant r_{0}$, where $r_{0}=\tanh \pi / 4$. This completes the proof of our theorem.

Robinson [8] was able to establish the relation (2) only for $|z|<1 / 5$. We have thus considerably improved his result.

The following result of Ruscheweyh and Sheil-Small [9, Theorem 4.1] finds an application in our next theorem.

LEMMA 2. Let $\phi$ and $\psi$ be convex in $|z|<1$ and suppose that $f$ is subordinate to $\psi$. Then $(\phi * f)(z) \prec(\phi * \psi)(z),|z|<1$. 
THEOREM 2. If $g(z)$ and $G(z)$ are regular for $|z|<1,[z G(z)]^{\prime}$ is univalent in $|z|<1$ and $[z g(z)]^{\prime}$ is subordinate to $[z G(z)]^{\prime}$ in $|z|<1$, then

$$
g(z) \prec G(z)
$$

at least for $|z|<2-\sqrt{3}=0.268 \ldots$

Proof. We define $f$ and $F$ by the relations

$$
2 f(z)=[z g(z)]^{\prime} \text { and } 2 F(z)=[z G(z)]^{\prime} .
$$

Thus we are given that

$$
2 f(z) \prec 2 F(z)
$$

or

$$
f(z) \prec F(z), \quad|z|<1 .
$$

We have to prove that

$$
g(z)=\frac{2}{z} \int_{0}^{z} f(t) d t \prec \frac{2}{z} \int_{0}^{z} F(t) d t=G(z),
$$

at least for $|z|<2-\sqrt{3}$.

Since the function $k(z)=z /(1-z)$ belongs to $K$, the function

$$
h(z)=\frac{2}{z} \int_{0}^{z} \frac{t}{1-t} d t=\sum_{n=1}^{\infty} \frac{2}{n+1} z^{n}
$$

also belongs to $K[4]$.

The function $F$ being univalent in $|z|<1$ is convex in the disc $|z|<2-\sqrt{3}=$ $r_{0}$ (say). Thus we are given that $f(z) \prec F(z)$ in $|z|<1$ and $F(z)$ is convex in $|z|<r_{0}$. From this we have that

$$
f\left(r_{0} z\right) \prec F\left(r_{0} z\right) \quad(|z|<1),
$$

with $F\left(r_{0} z\right)$ convex in $|z|<1$. In view of Lemma 2 it follows that

$$
h(z) * f\left(r_{0} z\right) \prec h(z) * F\left(r_{0} z\right)
$$

in $|z|<1$, which is the same as

$$
g\left(r_{0} z\right) \prec G\left(r_{0} z\right)
$$

in $|z|<1$ and hence

$$
g(z) \prec G(z)
$$

at least for $|z|<r_{0}=2-\sqrt{3}$.

It was earlier proved by Robinson [8] that the assertion (3) holds at least in the $\operatorname{disc}|z|<1 / 5$.

In the next theorem we will use the following result of Robertson [6] to determine a sufficient condition for a univalent function to be in $K(\alpha)$.

LEMMA 3. Let $f(z)=z+\sum_{n=2}^{\infty} a_{n} z^{n}$ be regular and univalent in $|z|<1$. For $0<t<1$ let $F(z, t)$ be regular in $|z|<1$. Let $F(z, 0) \equiv f(z)$ and $F(0, t) \equiv 0$. Let $p$ be a positive real number for which

$$
F(z)=\lim _{t \rightarrow 0+}\left[\frac{F(z, t)-F(z, 0)}{z t^{p}}\right]
$$


exists. Let $F(z, t)$ be subordinate to $f(z)$ in $|z|<1$ for $0<t<1$. Then

$$
\operatorname{Re}\left\{\frac{F(z)}{f^{\prime}(0)}\right\}<0, \quad|z|<1 .
$$

If in addition $F(z)$ is also analytic in $|z|<1$ and $\operatorname{Re} F(0) \neq 0$ then $\operatorname{Re}\left\{F(z) / f^{\prime}(z)\right\}$ $<0$ for $|z|<1$.

THEOREM 3. If $f$ belongs to $S$ and

$$
F(z, t)=\frac{1}{2}\left(1-\frac{i \alpha t}{2}\right) f\left(z e^{i t}\right)+\frac{1}{2}\left(1+\frac{i \alpha t}{2}\right) f\left(z e^{-i t}\right) \prec f(z)
$$

for $0<t<1,|z|<1$, and fixed real number $\alpha$, $\alpha<1$, then $f$ belongs to $K(\alpha)$.

Proof. Clearly $F(z, 0)=f(z), F(0, t)=0$. If

$$
F(z)=\lim _{t \rightarrow 0+}\left[\frac{F(z, t)-F(z, 0)}{z t^{2}}\right],
$$

then a simple calculation shows that

$$
\begin{aligned}
F(z) & =\lim _{t \rightarrow 0+}\left[\frac{\partial F(z, t) / \partial t}{2 z t}\right]=\lim _{t \rightarrow 0+}\left[\frac{1}{2 z} \frac{\partial^{2} F(z, t)}{\partial t^{2}}\right] \\
& =-\frac{1}{2}\left[z f^{\prime \prime}(z)+f^{\prime}(z)-\alpha f^{\prime}(z)\right] .
\end{aligned}
$$

Since $F(z)$ is regular in $|z|<1$ and $\operatorname{Re} F(0)=-(1-\alpha) / 2 \neq 0$, by the above lemma we have $\operatorname{Re}\left[F(z) / f^{\prime}(z)\right]<0$ and consequently

$$
\operatorname{Re}\left\{1+\frac{z f^{\prime \prime}(z)}{f^{\prime}(z)}\right\}>\alpha, \quad|z|<1,
$$

that is, $f \in K(\alpha)$.

REMARK. If $\alpha=0$ the subordination condition (5) becomes

$$
(1 / 2)\left[f\left(z e^{i t}\right)+f\left(z e^{-i t}\right)\right] \prec f(z)
$$

which is the result obtained by Robertson [0].

The following three theorems depend on a result due to Wilf [11] that characterizes subordinating factor sequences and, in particular, asserts that if

$$
\operatorname{Re}\left\{1+2 \sum_{n=1}^{\infty} b_{n} z^{n}\right\}>0 \text { for }|z|<1
$$

then $\left\{b_{n}\right\}$ is a subordinating factor sequence.

TheOrem 4. If $f$ belongs to $K$ and $g$ is defined by

$$
g(z)=\frac{\lambda}{z} \int_{0}^{z} f(t) d t,
$$

then for every $\lambda, 0<\lambda<1 / 2(1-\log 2)=1.629 \ldots$, we have

$$
g(z)<f(z) \quad(|z|<1),
$$

and this result is sharp. 
Proof. Suppose $f(z)=z+\sum_{n=2}^{\infty} a_{n} z^{n}$. Then

$$
g(z)=\frac{\lambda}{2} z+\sum_{n=2}^{\infty} \frac{\lambda}{n+1} a_{n} z^{n} .
$$

In view of Wilf's result, the assertion (9) will hold for $|z|<1$, if

$$
\operatorname{Re}\left\{1+2 \sum_{n=1}^{\infty} \frac{\lambda}{n+1} z^{n}\right\}>0 \quad(|z|<1)
$$

Using the fact that $\operatorname{Re}\{(-1 / z) \log (1-z)\}>\log 2$, [7], we have

$$
\begin{aligned}
\operatorname{Re}\left\{1+2 \sum_{n=1}^{\infty} \frac{\lambda}{n+1} z^{n}\right\} & =\operatorname{Re}\left\{(1-2 \lambda)-\frac{2 \lambda}{z} \log (1-z)\right\} \\
& >(1-2 \lambda)+2 \lambda \log 2>0
\end{aligned}
$$

if $\lambda<1 / 2(1-\log 2)$.

To show that this result is sharp we consider the function $f(z)=z(1-z)^{-1}$ which is an element of $K$. We have

$$
g(z)=\frac{\lambda}{z} \int_{0}^{z} \frac{t}{1-t} d t=\lambda\left\{-1-\frac{1}{z} \log (1-z)\right\} .
$$

Because $g(-1)=-\lambda\{1-\log 2\}<-1 / 2$ if $\lambda>1 / 2(1-\log 2)$ it is, therefore, not true that $g$ is subordinate to $f$ for $|z|<1$, since the range of $f$ is the half plane $\{w$ : $\operatorname{Re} w>-1 / 2\}$. The proof of Theorem 4 is, therefore, complete.

Theorem 4 with $\lambda=1$ was earlier proved by Bernardi [2].

REMARK. If $f$ belongs to $S$ and $g$ is defined as in (8) then, in view of the fact that $f$ is convex in $|z|<2-\sqrt{3}$, it follows that the subordination (9) will hold in $|z|<2-\sqrt{3}$ for all $\lambda, 0<\lambda<1 / 2(1-\log 2)$.

TheOrem 5. If $f$ belongs to $K$ and $g$ is defined by

$$
g(z)=\mu \int_{0}^{z} \frac{f(t)}{t} d t,
$$

then for every $\mu, 0<\mu<1 / 2 \log 2=0.721 \ldots$, we have

$$
g(z) \prec f(z) \quad(|z|<1) .
$$

The result is sharp.

Proof. In view of Wilf's result the assertion (12) will hold in $|z|<1$, if

$$
\operatorname{Re}\left\{1+2 \mu \sum_{n=1}^{\infty} \frac{1}{n} z^{n}\right\}>0 \quad(|z|<1) .
$$

Letting $z=r e^{i \theta}$, we find that

$$
\begin{aligned}
\operatorname{Re}\left\{1+2 \mu \sum_{n=1}^{\infty} \frac{1}{n} z^{n}\right\} & =\operatorname{Re}\{1-2 \mu \log (1-z)\} \\
& =\left\{1-2 \mu \log \left(1+r^{2}-2 r \cos \theta\right)^{1 / 2}\right\} \\
& >1-2 \mu \log 2>0
\end{aligned}
$$

provided $\mu<1 / 2 \log 2$. 
To show that the result is sharp we again consider the function $f(z)$ $=z(1-z)^{-1}$ which belongs to $K$. We have

$$
g(z)=-\mu \log (1-z) \text {. }
$$

Thus $g(-1)=-\mu \log 2<-1 / 2$, if $\mu>1 / 2 \log 2$ and so $g$ is not subordinate to $f$ for $|z|<1$ since the range of $f$ is the half plane $\{w: \operatorname{Re} w>-1 / 2\}$. This completes the proof of our theorem.

REMARK. If $f$ belongs to $S$ and $g$ is defined as in (11) then the assertion (12) holds in $|z|<2-\sqrt{3}$.

For $f(z)=\sum_{n=1}^{\infty} a_{n} z^{n}$, we define

(i) $s_{n}(z, f)=\sum_{k=1}^{n} a_{k} z^{k}$ and

(ii) $\sigma_{n}(z, f)=(1 / n) \sum_{k=1}^{n} s_{k}(z, f)$.

THEOREM 6. For all elements $f$ of $K$, we have

$$
\left(1 / \alpha_{n}\right) s_{n}(z, f)<f(z), \quad|z|<1,
$$

where $\alpha_{n}=-2 \min _{|z|<1} \operatorname{Re}\left\{\sum_{k=1}^{n} z^{k}\right\}$, and

$$
\left(1 / \beta_{n}\right) \sigma_{n}(z, f)<f(z), \quad|z|<1,
$$

where $\beta_{n}=-(2 / n) \min _{|z|<1} \operatorname{Re}\left\{\sum_{k=1}^{n}(n-k+1) z^{k}\right\}$.

Proof. Suppose

$$
f(z)=z+\sum_{n=2}^{\infty} a_{n} z^{n}
$$

then

$$
s_{n}(z, f)=z+a_{2} z^{2}+\cdots+a_{n} z^{n}
$$

and

$$
\sigma_{n}(z, f)=z+\frac{n-1}{n} a_{2} z^{2}+\frac{n-2}{n} a_{3} z^{3}+\cdots+\frac{1}{n} a_{n} z^{n} .
$$

In view of Wilf's result quoted above we shall have

$$
\left(1 / \alpha_{n}\right) s_{n}(z, f) \prec f(z), \quad|z|<1,
$$

if

$$
\operatorname{Re}\left\{1+\frac{2}{\alpha_{n}} \sum_{k=1}^{n} z^{k}\right\}>0, \quad|z|<1,
$$

which is true by the definition of $\alpha_{n}$.

This completes the proof of (13). Relation (14) may be similarly established.

RemarK. Since $\alpha_{n}<2 n$ and $\beta_{n}<n+1, n>1$, relations (13) and (14), in particular, show that

$$
(1 / 2 n) s_{n}(z, f) \prec f(z), \quad|z|<1,
$$

and

$$
(1 /(n+1)) \sigma_{n}(z, f) \prec f(z), \quad|z|<1 .
$$

The well-known result $z / 2 \prec f(z)$ for every $f$ belonging to $K$ corresponds to $n=1$ in (13) and (14). 
It is easy to compute that $\alpha_{2}=9 / 4$ and $\beta_{2}=3 / 2$. Thus for $n=2$ the above theorem gives

and

$$
(4 / 9) s_{2}(z, f) \prec f(z), \quad|z|<1,
$$

$$
(2 / 3) \sigma_{2}(z, f) \prec f(z), \quad|z|<1 .
$$

We will need the following lemma due to Keogh [3] to prove our next theorem.

LEMmA 4. Suppose that $b_{0}, b_{1}, b_{2}$ are complex numbers, $b_{2} \neq 0$, and let $P(z)=b_{0}$ $+b_{1} z+b_{2} z^{2}$. Then the zeros of $P(z)$ lie on $|z|<1$ if, and only if

(i) $\left|b_{0}\right| \leqslant\left|b_{2}\right|$ and

(ii) $\left|b_{0} \bar{b}_{1}-b_{1} \bar{b}_{2}\right| \leqslant\left|b_{2}\right|^{2}-\left|b_{0}\right|^{2}$.

THEOREM 7. If $f$ belongs to $K$, then we have

$$
V_{2}(z, f) \prec s_{2}(z, f)
$$

in $|z|<2 / 5$, and this result is sharp;

in $|z|<1$.

$$
z / 2 \prec V_{2}(z, f) \prec \sigma_{2}(z, f)
$$

Proof. It is well known that $s_{2}(z, f)$ is starlike univalent in $|z|<1 / 2$ and convex in $|z|<1 / 4$, and that $V_{2}(z, f)$ is convex for every convex function $f$ in $|z|<1$.

First, we want to prove that $V_{2}(z, f)$ is subordinate to $s_{2}(z, f)$ in $|z|<2 / 5=r_{0}$ (say). That is, $V_{2}\left(r_{0} z, f\right) \prec s_{2}\left(r_{0} z, f\right)$ in $|z|<1$. Following BaşGöze et al. [1], it is sufficient to show that for each real $\alpha$, the polynomial

$$
P(z)=a_{2} r_{0}^{2} z^{2}+r_{0} z-\left(\frac{2}{3} r_{0} e^{i \alpha}+\frac{1}{6} a_{2} r_{0}^{2} e^{2 i \alpha}\right)
$$

has a zero on $|z| \leqslant 1$. Suppose that for some $\alpha$ it has no zero in $|z|<1$. Then the polynomial

$$
Q(z)=\left(\frac{2}{3} r_{0} e^{i \alpha}+\frac{1}{6} a_{2} r_{0}^{2} e^{2 i \alpha}\right) z^{2}-r_{0} z-a_{2} r_{0}^{2}
$$

has both zeros on $|z|<1$; hence by Lemma 4,

$$
\left|a_{2} r_{0}^{3}+r_{0}\left(\frac{2}{3} r_{0} e^{-i \alpha}+\frac{1}{6} \bar{a}_{2} r_{0}^{2} e^{-2 i \alpha}\right)\right| \leqslant\left|\frac{2}{3} r_{0} e^{i \alpha}+\frac{1}{6} a_{2} r_{0}^{2} e^{2 i \alpha}\right|^{2}-\left|a_{2} r_{0}^{2}\right|^{2} .
$$

Writing $a_{2} r_{0}=\rho e^{i \phi}, \alpha+\phi=\psi$, this is equivalent to

$$
\left|4+\rho e^{i \psi}\right|^{2}-6\left|6 \rho e^{i \psi}+4+\rho e^{-i \psi}\right|>36 \rho^{2} .
$$

One readily verifies that the maximum of the left-hand side of (19) is attained at $\psi=\pi$. Also for $\psi=\pi$ the inequality (i) of Lemma 4 implies that $\rho<4 / 7$. Therefore (19) will fail to hold if $\rho^{2}+34 \rho-8<36 \rho^{2}$ or

$$
(\rho-2 / 5)(\rho-4 / 7)>0 \text {. }
$$

Since $\rho \leqslant 4 / 7$, the inequality (19) will not hold for all $\rho<2 / 5$. This proves that the polynomial $P(z)$ has all its zeros in $|z|<1$ for $\rho<2 / 5$. Since $\rho=\left|a_{2}\right| r_{0}<r_{0}$, we have proved that for $r_{0}<2 / 5, P(z)$ has all its zeros in $|z|<1$ and hence

$$
V_{2}\left(r_{0} z, f\right)<s_{2}\left(r_{0} z, f\right) \text { in }|z|<1\left(r_{0}<2 / 5\right) \text {. }
$$


This establishes the relation

$$
V_{2}(z, f)<s_{2}(z, f) \text { in }|z|<r_{0} .
$$

To show that our result is sharp in the sense that if $2 / 5<r<1$ then there is a function $f$ in $K$ so that $V_{2}(z, f)$ is not subordinate to $s_{2}(z, f)$ for $|z|<r$, we consider the function $f(z)=z /(1-z)$. Then $V_{2}(z, f)=(2 / 3) z+(1 / 6) z^{2}$ and $s_{2}(z, f)=z$ $+z^{2}$. Since $V_{2}(-2 / 5, f)=s_{2}(-2 / 5, f), V_{2}(z, f)$ is not subordinate to $s_{2}(z, f)$ for $|z|<r$ (if $2 / 5<r<1$ ). The last assertion depends upon the strict sense in which equality is possible in Lindelöf's Principle for subordination.

As for the proof of (16) we remark that since $\sigma_{2}(z, f)$ is univalent (in fact starlike) in $|z|<1$ and the relation $z / 2 \prec V_{2}(z, f)$ for every $f$ belonging to $K$ being well known, we need to prove only

$$
V_{2}(z, f)<\sigma_{2}(z, f), \quad|z|<1 .
$$

It suffices to show that for each real $\alpha$, the polynomial

$$
R(z)=z+\frac{1}{2} a_{2} z^{2}-\frac{2}{3} e^{i \alpha}-\frac{1}{6} a_{2} e^{2 i \alpha}
$$

has a zero on $|z| \leqslant 1$. Suppose that for some $\alpha$ it has no zero in $|z|<1$. Then the polynomial

$$
T(z)=\left(\frac{2}{3} e^{i \alpha}+\frac{1}{6} a_{2} e^{2 i \alpha}\right) z^{2}-z-\frac{1}{2} a_{2}
$$

has both zeros on $|z| \leqslant 1$; hence by Lemma 5 ,

$$
\left|\frac{1}{2} a_{2}+\frac{2}{3} e^{-i \alpha}+\frac{1}{6} \bar{a}_{2} e^{-2 i \alpha}\right|<\left|\frac{2}{3} e^{i \alpha}+\frac{1}{6} a_{2} e^{2 i \alpha}\right|^{2}-\left|\frac{1}{2} a_{2}\right|^{2} .
$$

Writing $a_{2}=\rho e^{i \phi}, \alpha+\phi=\delta$, this is equivalent to

$$
\left|4+\rho e^{i \delta}\right|^{2}-6\left|3 \rho e^{i \delta}+4+\rho e^{-i \delta}\right|>9 \rho^{2} .
$$

Proceeding as in the proof of (15) we arrive at the conclusion that for $\rho<1$, (23) is not true and hence $R(z)$ will have all its zeros in $|z|<1$ for $\rho<1$. This then will complete the proof of (16).

ACKNOWLEDGEMENT. The authors wish to thank the referee for his many valuable suggestions and comments.

\section{REFERENCES}

1. T. BaşGöze, J. L. Frank and F. R. Keogh, On convex univalent functions, Canad. J. Math. 22 (1970), 123-127.

2. S. D. Bernardi, Special classes of subordinate functions, Duke Math. J. 33 (1966), 55-67.

3. F. R. Keogh, A strengthened form of the $\frac{1}{4}$-theorem for starlike and univalent functions, Mathematical Essays Dedicated to A. J. MacIntyre, Ohio Univ. Press, Athens, Ohio (to appear).

4. R. J. Libera, Some classes of regular univalent functions, Proc. Amer. Math. Soc. 16 (1965), 755-758.

5. G. Pólya and I. J. Schoenberg, Remarks on de la Vallée Poussin means and convex conformal maps of the circle, Pacific J. Math. 8 (1958), 295-334.

6. M. S. Robertson, Application of subordination principle to univalent functions, Pacific J. Math. 11 (1961), 315-324.

7. _ـ An extremal problem for functions with positive real part, Michigan Math. J. 11 (1964). 
8. R. M. Robinson, Univalent majorants, Trans. Amer. Math. Soc. 61 (1947), 1-35.

9. St. Ruscheweyh and T. Sheil-Small, Hadamard products of schlicht functions and the Pólya-Schoenberg conjecture, Comment. Math. Helv. 48 (1973), 119-135.

10. T. J. Suffridge, Some remarks on convex maps of the unit disc, Duke Math. J. 37 (1970), 775-777.

11. H. S. Wilf, Subordinating factor sequences for convex maps of the unit circle, Proc. Amer. Math. Soc. 12 (1961), 689-693.

Department of Mathematics, Punjabi University, Patiala (PB), India (Current address of Ram Singh)

Current address (Sunder Singh): Department of Mathematics, Government College for Women, Srinagar (Jammu and Kashmir), India 